\title{
ON CONSTRUCTING DISTRIBUTION FUNCTIONS; WITH APPLICATIONS TO LOMMEL POLYNOMIALS AND BESSEL FUNCTIONS
}

\author{
BY \\ DANIEL MAKI
}

Given two sequences of real numbers, $\left\{a_{n}\right\}_{0}^{\infty}$ and $\left\{b_{n}\right\}_{1}^{\infty}$, where the $b_{n}$ 's are all positive, then by a result of Favard [12], there exists a bounded increasing function $\psi(x)$ defined on $(-\infty,+\infty)$ and having the following property:

$$
\int_{-\infty}^{+\infty} \phi_{n}(x) \phi_{m}(x) d \psi(x)=\delta_{n, m} \cdot k_{n}, \quad k_{n} \neq 0, n=0,1,2, \ldots
$$

where the polynomials $\phi_{n}(x)$ are recursively defined by: $\phi_{-1}(x)=0, \phi_{0}(x)=1$, and

$$
\phi_{n+1}(x)=\left(x-a_{n}\right) \phi_{n}(x)-b_{n} \phi_{n-1}(x) \quad(n \geqq 0) .
$$

This study begins by showing how to obtain such a function $\psi(x)$ for certain classes of sequences $\left\{a_{n}\right\}_{0}^{\infty}$ and $\left\{b_{n}\right\}_{1}^{\infty}$. Then we apply our results to obtain a distribution function for the modified Lommel polynomials (thus answering a question of Dickinson, [10, p. 121]) and to obtain some information about Bessel functions as a function of their order.

2. Notation, preliminaries, and summary. The following notational conventions will be maintained throughout this paper:

(1) $\left\{a_{n}\right\}_{0}^{\infty}$ is a sequence of real numbers.

(2) $\left\{b_{n}\right\}_{1}^{\infty}$ is a sequence of positive real numbers.

For each nonnegative integer $s$,

(3) $\left\{c_{n}^{(s)}\right\}_{0}^{\infty}$ is the sequence $\left\{c_{n+s}\right\}_{n=0}^{\infty}$.

(4) $\left\{\phi_{n}^{(s)}(x)\right\}_{-1}^{\infty}$ is the sequence of monic polynomials defined recursively by $\phi_{-1}^{(s)}(x)=0, \phi_{0}^{(s)}(x)=1$, and $\phi_{n+1}^{(s)}(x)=\left(x-a_{n}^{(s)}\right) \phi_{n}^{(s)}(x)-b_{n}^{(s)} \phi_{n-1}^{(s)}(x)(n \geqq 0)$.

(5) $\psi^{(s)}(x)$ is a bounded increasing function defined on $(-\infty,+\infty)$ and having the property that

$$
\int_{-\infty}^{+\infty} \phi_{n}^{(s)}(x) \phi_{m}^{(s)}(x) d \psi^{(s)}(x)=\delta_{n, m} \cdot k_{n} \quad\left(k_{n} \neq 0, n=0,1,2, \ldots\right) .
$$

$\left(\psi^{(s)}(x)\right.$ is known to exist, by the above-mentioned theorem of Favard.)

(6) $K^{(s)}(x)$ is the continued fraction given by

$$
K^{(s)}(x)=\frac{1 \mid}{\mid x-a_{s}}-\frac{b_{1+s} \mid}{\mid x-a_{1+s}}-\frac{b_{2+s} \mid}{\mid x-a_{2+s}}-\cdots
$$

Received by the editors June 2, 1966. 
(7) $\mathscr{S}\left(\psi^{(s)}(x)\right)$ is the spectrum of the distribution function $\psi^{(s)}(x)$, i.e., $\mathscr{S}\left(\psi^{(s)}(x)\right)$ $=\left\{x:-\infty<x<+\infty\right.$ and $\psi^{(s)}(x+\varepsilon)-\psi^{(s)}(x-\varepsilon)>0$ for all $\left.\varepsilon>0\right\}$. In terms of measures, $\mathscr{S}\left(\psi^{(s)}(x)\right)$ is the support of the positive real measure induced by $\psi^{(s)}(x)$.

(8) We shall say that the polynomials $\phi_{n}^{(s)}(x)$, the bounded increasing function $\psi^{(s)}(x)$, and the continued fraction $K^{(s)}(x)$ are associated with the sequences $\left\{a_{n}\right\}_{0}^{\infty}$ and $\left\{b_{n}\right\}_{1}^{\infty}$ if they are related to these sequences by (4), (5), and (6) above.

(9) $C$ will represent the field of complex numbers.

In terms of the techniques which are used, this study is a continuation of the work of Dickinson, Pollak and Wannier [11], and that of Goldberg [14]. It differs from these papers in considering unbounded sequences and hence requires some additional tools. Chihara has also considered this problem and by using the theory of chain sequences has obtained a number of theorems dealing with properties of $\mathscr{S}\left(\psi^{(s)}(x)\right)$. We will use one particular result of his and quote it now for reference.

TheOrem 2.1 (ChinARA [6, p. 4]). A necessary and sufficient condition for the polynomials $\phi_{n}^{(s)}(x)$ to be orthogonal over an interval which is a subset of $[0, \infty)$ is that $a_{n}>0$ for each $n$ and $\left\{b_{n} /\left(a_{n} \cdot a_{n-1}\right)\right\}$ be a chain sequence.

We will also need some well-known results from the general theory of orthogonal polynomials. These can all be found in Szegö's book [17]. We collect them into the following lemma.

LEMMA 2.2. The convergents of the continued fraction $K^{(s)}(x)$ are the rational functions $\phi_{n-1}^{(s+1)}(x) / \phi_{n}^{(s)}(x)$, and the zeros of the monic polynomials $\phi_{n}^{(s)}(x)$ are real, simple, and interlaced with the zeros of $\phi_{n-1}^{(s+1)}(x)$.

We now enumerate those conditions which we will impose upon the sequences $\left\{a_{n}\right\}_{0}^{\infty}$ and $\left\{b_{n}\right\}_{1}^{\infty}$. These are as follows:

(1) $a_{i} \rightarrow \infty$ as $i \rightarrow \infty$.

(2) $\lim \sup _{n \rightarrow \infty} b_{i} /\left(a_{i} \cdot a_{i-1}\right)=L<1 / 4$.

Under these conditions we shall show that $K^{(s)}(x)$ is meromorphic and $\psi^{(s)}(x)$ can always be chosen to be a jump function with jumps at the poles of $K^{(s)}(x)$.

3. $K^{(s)}(x)$ is meromorphic. In this section we show that $K^{(s)}(x)$ is a meromorphic function, and we give a Mittag-Leffler expansion for it. To do this we use the following continued fraction theorem:

THEOREM 3.1 (WORPITSKY [19, p. 42]). Let $a_{2}, a_{3}, \ldots$ be complex functions of any variables over a domain $D$ in which $\left|a_{p+1}\right| \leqq 1 / 4, p=1,2, \ldots$ Then the following statements hold:

(i) The continued fraction

$$
w=\frac{1 \mid}{\mid 1}+\frac{a_{2} \mid}{\mid 1}+\frac{a_{3} \mid}{\mid 1}+\cdots
$$

converges uniformly over $D$. 
(ii) The values of the continued fraction and of its approximates are in the circular domain

$$
|w-4 / 3| \leqq 2 / 3 \text {. }
$$

Using this we now prove our first result.

THEOREM 3.2. Let the sequences $\left\{a_{n}\right\}_{0}^{\infty}$ and $\left\{b_{n}\right\}_{1}^{\infty}$ satisfy the conditions:

(1) $a_{n} \rightarrow \infty$ as $n \rightarrow \infty$.

(2) $\lim _{\sup } \rightarrow \infty b_{n} /\left(a_{n} \cdot a_{n-1}\right)=L<1 / 4$.

Then the continued fraction $K^{(s)}(x)$ defined by (6) of $\$ 2$ is a meromorphic function.

Proof. By definition

$$
K^{(s)}(x)=\frac{1 \mid}{\mid x-a_{s}}-\frac{b_{1+s} \mid}{\mid x-a_{1+s}}-\frac{b_{2+s} \mid}{\mid x-a_{2+s}}-\cdots ;
$$

so by an equivalence transformation of this continued fraction we have

$$
\begin{aligned}
K^{(s)}(x)=\frac{1 /\left(x-a_{s}\right) \mid}{\mid 1}-\frac{b_{1+s} /\left(x-a_{1+s}\right)\left(x-a_{s}\right) \mid}{\mid 1} & -\frac{b_{2+s} /\left(x-a_{1+s}\right)\left(x-a_{2+s}\right) \mid}{\mid}-\cdots .
\end{aligned}
$$

Now let $D$ be any bounded domain. Then, since $a_{i} \rightarrow \infty$ and $L<1 / 4$, we know there exists an integer $N$, which depends on $D$, such that for $n>N$ and $x \in D$ we have

$$
\begin{aligned}
\left|\frac{b_{n}}{\left(x-a_{n}\right)\left(x-a_{n-1}\right)}\right| & =\left|\frac{b_{n}}{a_{n} \cdot a_{n-1}}\right| \cdot\left|\frac{1}{\left(1-x / a_{n}\right)\left(1-x / a_{n-1}\right)}\right| \\
& \leqq 1 / 4 .
\end{aligned}
$$

Thus by Worpitsky's theorem the continued fraction

$$
K_{N}(x)=\frac{1 \mid}{\mid 1}-\frac{b_{N} /\left(x-a_{N}\right)\left(x-a_{N-1}\right) \mid}{\mid 1}-\frac{b_{N+1}\left|\left(x-a_{N+1}\right)\left(x-a_{N}\right)\right|}{\mid 1}-\cdots
$$

converges uniformly in the domain $D$. Also, by (ii) of Worpitsky's theorem, the convergents of $K_{N}(x)$, which are rational functions, do not have any poles in $D$ and hence are actually analytic in $D$. Thus because of the uniform convergence $K_{N}(x)$ is also analytic in $D$. But $K_{N}(x)$ is just the tail end of $K^{(s)}(x)$ and thus $K^{(s)}(x)$ must be meromorphic in $D$ with uniform convergence on compact sets which exclude poles. Moreover $D$ was any bounded domain, so $K^{(s)}(x)$ is meromorphic in $C$. Q.E.D.

We next give a Mittag-Leffler expansion for $K^{(s)}(x)$. For this we need a theorem of Montel, which we now quote.

THeOREM 3.3 (MONTEL [15, p. 42]). A necessary and sufficient condition for a meromorphic function $G(z)$ to be the uniform limit of rational functions whose zeros and poles are interlaced on the positive real axis is that $G(z)$ have the form

$$
G(z)=-A+\sum_{1}^{\infty} A_{i} /\left(z-\alpha_{i}\right)
$$

where $A$ and all $A_{i}$ 's are real and of the same sign and $\sum_{1}^{\infty} A_{i} / \alpha_{i}$ converges. 
Using Theorem 3.3 we now prove

THEOREM 3.4. The meromorphic function $K^{(s)}(x)$ has the following Mittag-Leffler expansion:

$$
K^{(s)}(x)=-A^{(s)}+\sum_{1}^{\infty} A_{i}^{(s)} /\left(x-\alpha_{i}^{(s)}\right)
$$

where $A^{(s)}$ and $A_{i}^{(s)}$ are real, $A^{(s)}$ and $A_{i}^{(s)}$ are of the same sign, $i=1,2, \ldots$, and $\sum_{1}^{\infty} A_{i}^{(s)} / \alpha_{i}^{(s)}$ converges.

Proof. By Lemma 2.2 we have

$$
K^{(s)}(x)=\lim _{m \rightarrow \infty} \phi_{m-1}^{(s+1)}(x) / \phi_{m}^{(s)}(x),
$$

where the zeros and poles of the rational functions $\phi_{m-1}^{(s+1)}(x) / \phi_{m}^{(s)}(x)$ are interlaced on the real axis. Now, from the general theory of chain sequences, (see $[6, \mathrm{pp}$. $1-4]$ ), we know that conditions (1) and (2) above imply the existence of a constant $c \geqq 0$ such that the sequences $\left\{a_{n}^{*}=a_{n}+c\right\}_{0}^{\infty}$ and $\left\{b_{n}\right\}_{1}^{\infty}$ satisfy

(a) $a_{n}^{*}>0$ for each $n$,

(b) $a_{n}^{*} \rightarrow \infty$ as $n \rightarrow \infty$,

(c) $\left\{b_{n} /\left(a_{n}^{*} \cdot a_{n-1}^{*}\right)\right\}$ is a chain sequence.

Thus by Theorem 2.1 we know that any distribution $\psi_{*}^{(s)}(x)$ associated with the sequences $\left\{a_{n+s}^{*}\right\}$ and $\left\{b_{n+s}\right\}$ has its spectrum in $[0, \infty)$. But if $\psi_{*}^{(s)}(x)$ is a distribution associated with $\left\{a_{n+s}+c\right\}_{n=0}^{\infty}$ and $\left\{b_{n}^{(s)}\right\}_{1}^{\infty}$, then $\psi^{(s)}(x)=\psi_{*}^{(s)}(x-c)$ is a distribution associated with $\left\{a_{n}^{(s)}\right\}_{n=0}^{\infty}$ and $\left\{b_{n}^{(s)}\right\}_{n=1}^{\infty}$, and conversely. Thus we can assume $\mathscr{S}\left(\psi^{(s)}(x)\right) \subset(-c, \infty)$ for some finite $c \geqq 0$. Next, the zeros of the polynomials $\phi_{n}^{(s)}(x)$ are always contained in the same interval as the spectrum of their distribution function and hence the rational functions $\phi_{m-1}^{(s+1)}(x) / \phi_{m}^{(s)}(x)$ must have their zeros and poles interlaced on the interval $(-c, \infty)$. Thus by a direct application of Theorem 3.3 to the function $K^{(s)}(x-c)$ the result follows. Q.E.D.

We next prove a result about the behavior of $x K^{(s)}(x)$ for large imaginary $x$.

LEMMA 3.5. For y real

$$
\lim _{y \rightarrow \infty}(i y) K^{(s)}(i y)=1
$$

Proof. By definition

$$
\begin{aligned}
x K^{(s)}(x) & =\frac{x /\left(x-a_{s}\right) \mid}{\mid 1}-\frac{b_{1+s}\left|\left(x-a_{s}\right)\left(x-a_{1+s}\right)\right|}{1} \\
& -\frac{b_{2+s}\left|\left(x-a_{1+s}\right)\left(x-a_{2+s}\right)\right|}{\mid}-\cdots \\
& =\left\{\frac{x}{x-a_{s}}\right\} \frac{1}{1-\frac{b_{1+s}}{\left(x-a_{s}\right)\left(x-a_{s+1}\right)}\left\{\frac{1 \mid}{\mid 1}-\frac{b_{2+s}\left|\left(x-a_{s+2}\right)\left(x-a_{s+1}\right)\right|}{\mid}-\cdots\right\}} .
\end{aligned}
$$


Now for $x=i y, x /\left(x-a_{s}\right) \rightarrow 1$ as $y \rightarrow \infty$. Also $b_{1+s} /\left(x-a_{s}\right)\left(x-a_{s+1}\right) \rightarrow 0$, and by Worpitsky's theorem (Theorem 3.1 above) the continued fraction

$$
\frac{1 \mid}{\mid 1}-\frac{b_{2+s}\left|\left(x-a_{1+s}\right)\left(x-a_{2+s}\right)\right|}{\mid}-\frac{b_{3+s}\left|\left(x-a_{2+s}\right)\left(x-a_{3+s}\right)\right|}{\mid}-\cdots
$$

will remain bounded for $x=i y$ and $y$ large. Therefore, for $x=i y, x K^{(s)}(x) \rightarrow 1$ as $y \rightarrow \infty$. Q.E.D.

Corollary 3.6. $K^{(s)}(x)=\sum_{1}^{\infty} A_{i}^{(s)} /\left(x-\alpha_{i}^{(s)}\right)$.

Proof. From Theorem 3.4 we have

$$
K^{(s)}(x)=-A^{(s)}+\sum_{1}^{\infty} A_{i}^{(s)} /\left(x-\alpha_{i}^{(s)}\right) .
$$

Now from Lemma 3.5 it follows that $\lim _{y \rightarrow \infty} K^{(s)}(i y)=0$, so if we can show that $\lim _{y \rightarrow \infty} K^{(s)}(i y)=-A^{(s)}$, the corollary will follow. Let $\varepsilon>0$ be given. Then

$$
\begin{aligned}
\left|K^{(s)}(i y)+A^{(s)}\right| & =\left|\sum_{k=1}^{\infty} A_{k}^{(s)} /\left(i y-\alpha_{k}^{(s)}\right)\right| \\
& \leqq \sum_{k=1}^{K} \frac{A_{k}^{(s)}}{\left|i y-\alpha_{k}^{(s)}\right|}+\sum_{k=K+1}^{\infty} \frac{A_{k}^{(s)}}{\alpha_{k}^{(s)}} \frac{1}{|y| \alpha_{k}^{(s)}-i \mid} \\
& \leqq \sum_{k=1}^{K} \frac{A_{k}^{(s)}}{|y|}+\sum_{k=K+1}^{\infty} \frac{A_{k}^{(s)}}{\alpha_{k}^{(s)}} .
\end{aligned}
$$

Now by the interlacing of the zeros of $\phi_{n}^{(s)}(x)$ with those of $\phi_{n-1}^{(s+1)}(x)$ and by Theorem 3.4 each $A_{k}^{(s)}$ is positive and $\sum_{1}^{\infty} A_{k}^{(s)} / \alpha_{k}^{(s)}$ converges. Hence we first choose $K$ large enough so that $\sum_{k=K+1}^{\infty} A_{k}^{(s)} / \alpha_{k}^{(s)}<\varepsilon / 2$ and then choose $y$ large enough so that $\left(\sum_{k=1}^{K} A_{k}^{(s)}\right)(1 /|y|)<\varepsilon / 2$. This gives $\left|K^{(s)}(i y)+A^{(s)}\right|<\varepsilon$ and proves the corollary, since $\varepsilon$ was arbitrary. Q.E.D.

COROLlaRY 3.7. For $0 \leqq p \leqq n$ we have

$$
\lim _{y \rightarrow \infty}(i y)^{p+1} K^{(s)}(i y) \cdots K^{(s+n)}(i y)=\delta_{n, p} .
$$

Proof. This follows directly from Lemma 3.5.

4. Constructing $\psi^{(s)}(x)$. At this point it would be possible to construct $\psi^{(s)}(x)$ by using a number of theorems which deal with the Hamburger Moment Problem. We choose to use another, somewhat longer approach because it gives us new information about all sets of orthogonal polynomials whose associated sequences satisfy (1) and (2) above. We begin by quoting a special case of a Dickinson result.

LEMMA 4.1 [10, p. 199]. For $s \geqq 0, n \geqq 1$, and $m \geqq n+1$ we have

$$
b_{s+n} \phi_{n-1}^{(s)}(x) \phi_{m-n-1}^{(s+n+1)}(x)=\phi_{n}^{(s)}(x) \phi_{m-n}^{(s+n)}(x)-\phi_{m}^{(s)}(x) .
$$


Next, before proceeding with other lemmas, we make the following convention regarding the zeros of the polynomials $\phi_{m}^{(s)}$ : for each $s \geqq 0$ and $m \geqq 1$ we let $\left\{\alpha_{i, m}^{(s)}\right\}_{i=1}^{m}$ be the zeros of $\phi_{m}^{(s)}(x)$ ordered so that $\alpha_{1, m}^{(s)}<\alpha_{2, m}^{(s)}<\cdots<\alpha_{m, m}^{(s)}$. Also since $K^{(s)}(x)$ is meromorphic with all its poles in $(-c, \infty), c \geqq 0$, it has only finitely many poles in the interval $\left(-c, \alpha_{n, n}^{(s)}\right)$ for each fixed $n \geqq 1$ and $s \geqq 0$. Let this number be $N(n, s)$. We now continue with our results.

LEMMA 4.2. For each $n \geqq 1$ and $s \geqq 0$, there exists an integer $M(n, s)$ with the property that $m>M(n, s)$ implies $\phi_{m}^{(s)}(x)$ has exactly $N(n, s)$ zeros in the interval $\left(-c, \alpha_{n, n}^{(s)}\right)$.

Proof. From Theorem 3.4 we know that the poles of $K^{(s)}(x)$ are simple. Hence from Lemma 2.2 and Hurwitz's theorem we deduce that in small neighborhoods of each pole of $K^{(s)}(x), \phi_{m-1}^{(s+1)}(x) / \phi_{m}^{(s)}(x)$ eventually has one pole and no zeros. Likewise in compact sets free of poles $\phi_{m-1}^{(s+1)}(x) / \phi_{m}^{(s)}(x)$ is eventually free of poles. Therefore, since there are $N(n, s)$ poles of $K^{(s)}(x)$ in $\left(-c, \alpha_{n, n}^{(s)}\right)$, for sufficiently large $m, \phi_{m}^{(s)}(x)$ has one zero near each of these poles and no other zeros in this compact set. Q.E.D.

Our next result deals with the interlacing of the zeros of $\phi_{m-n-1}^{(s+n+1)}(x)$ with those of $\phi_{m}^{(s)}(x)$. These zeros do not interlace on the whole real axis, however for fixed $s \geqq 0$ and $n \geqq 0$ we can show that for all large $m$ those zeros in the interval $\left(\alpha_{n, n}^{(s)}, \infty\right)$ are interlaced.

LEMMA 4.3. For each fixed $n \geqq 0$ and $s \geqq 0$, there exists an integer $M^{*}(n, s)$ with the property that if $m>M^{*}(n, s)$, then for $m>i>N(n, s) \phi_{m-n-1}^{(s+n+1)}(x)$ has exactly one zero in the interval $\left(\alpha_{i, m}^{(s)}, \alpha_{i+1, m}^{(s)}\right)$.

Proof. We use induction on $n$. For $n=0$ and $s \geqq 0$ we know by Lemma 2.2 that $\phi_{m-1}^{(s+1)}(x)$ and $\phi_{m}^{(s)}(x)$ have interlacing zeros on the whole real axis whenever $m \geqq 2$. Thus we can choose $M^{*}(0, s)=2$. Next consider the general case and assume that the lemma holds for $n=k-1$, and $s \geqq 0$. Then consider $n=k$. From Lemma 4.1 we have

$$
(\mathrm{IV}-\mathrm{A}) \quad b_{s+k} \phi_{k-1}^{(s)}(x) \phi_{m-k-1}^{(s+k+1)}(x)=\phi_{k}^{(s)}(x) \phi_{m-k}^{(s+k)}(x)-\phi_{m}^{(s)}(x) \quad(m \geqq k+1),
$$

and from Lemma 4.2 we know that there exists an integer $M(k, s)$ such that $m>M(k, s)$ implies $\phi_{m}^{(s)}(x)$ has exactly $N(k, s)$ zeros in $\left(-c, \alpha_{k, k}^{(s)}\right)$. Also we know from Lemma 2.2 that all the zeros of $\phi_{k}^{(s)}(x)$ and $\phi_{k-1}^{(s+1)}(x)$ are contained in the interval $\left(-c, \alpha_{k, k}^{(s)}\right)$. Thus for $m>\max \left\{M(k, s), M^{*}(k-1, s), N(k, s)\right\}$ and $m>i>N(k, s)$ we know that the monic polynomials $\phi_{k}^{(s)}(x)$ and $\phi_{k-1}^{(s+1)}(x)$ are positive in $\left(\alpha_{i, m}^{(s)}, \alpha_{i+1, m}^{(s)}\right)$. Since $b_{s+k}>0$ and $\alpha_{i, m}^{(s)}, \alpha_{i+1, m}^{(s)}$ are zeros of $\phi_{m}^{(s)}(x)$ we deduce from (IV-A) that

$$
\begin{gathered}
\operatorname{sign} \phi_{m-k-1}^{(s+k+1)}\left(\alpha_{i, m}^{(s)}\right)=\operatorname{sign} \phi_{m-k}^{(s+k)}\left(\alpha_{i, m}^{(s)}\right), \\
\operatorname{sign} \phi_{m-k-1}^{(s+k+1)}\left(\alpha_{i+1, m}^{(s)}\right)=\operatorname{sign} \phi_{m-k}^{(s+k)}\left(\alpha_{i+1, m}^{(s)}\right) .
\end{gathered}
$$


Now $m>M^{*}(k-1, s)$ and $m>i>N(k, s)$ so by the induction hypothesis $\phi_{m-k}^{(s+k)}(x)$ has exactly one zero in $\left(\alpha_{i, m}^{(s)}, \alpha_{i+1, m}^{(s)}\right)$. Combining this with (IV-B) we see that $\phi_{m-k-1}^{(s+k+1)}(x)$ must have an odd number of zeros in $\left(\alpha_{i, m}^{(s)}, \alpha_{i+1, m}^{(s)}\right)$. However if this number is three or more, then by the known interlacing of the zeros of $\phi_{m-k}^{(s+k)}(x)$ with those of $\phi_{m-k-1}^{(s+k+1)}(x), \phi_{m-k}^{(s+k)}(x)$ will have at least two zeros in $\left(\alpha_{i, m}^{(s)}, \alpha_{i+1, m}^{(s)}\right)$ and this will contradict the induction hypothesis. Hence $\phi_{m-k-1}^{(s+k+1)}(x)$ has exactly one zero in $\left(\alpha_{i, m}^{(s)}, \alpha_{i+1, m}^{(s)}\right)$ and the lemma follows by induction taking $M^{*}(0, s)=2$ and $M^{*}(k, s)=\max \left\{M(k, s), M^{*}(k-1, s), N(k, s)\right\}, k \geqq 1$.

Using the above lemmas we now obtain a partial fraction expansion for the rational function $x^{p} \phi_{m-n-1}^{(s+n+1)}(x) / \phi_{m}^{(s)}(x)$.

COROLlaRY 4.4. For $s \geqq 0,0 \leqq p \leqq n$ and $m>M^{*}(n, s)$ we have

$$
\frac{x^{p} \phi_{m-n-1}^{(s+n+1)}(x)}{\phi_{m}^{(s)}(x)}=\sum_{i=1}^{m} \frac{B^{(s)}(i, m ; p, n)}{x-\alpha_{i, m}^{(s)}}
$$

where $B^{(s)}(i, m ; p, n)>0$ for $N(n, s)<i \leqq m$.

Proof. Since $\phi_{m}^{(s)}(x)=\prod_{i=1}^{n}\left(x-\alpha_{i, m}^{(s)}\right)$ where $\alpha_{1, m}^{(s)}<\alpha_{2, m}^{(s)}<\cdots<\alpha_{m, m}^{(s)}$, an expansion of the desired type exists; and we need only establish the condition on the residues $B^{(s)}(i, m ; p, n)$. By standard techniques these residues are given by

$$
B^{(s)}(i, m ; p, m)=\left(\alpha_{i, m}^{(s)}\right)^{p} \frac{\prod_{j=1}^{m-n-1}\left(\alpha_{i, m}^{(s)}-\alpha_{j, m-n-1}^{(s+n+1)}\right)}{\prod_{j=1 ; j \neq i}^{m}\left(\alpha_{i, m}^{(s)}-\alpha_{j, m}^{(s)}\right)} .
$$

Now by Lemma 4.3, we know that for $m>M^{*}(n, s)$ those zeros of $\phi_{m-n-1}^{(s+n+1)}(x)$ and $\phi_{m}^{(s)}(x)$ which are greater than $\alpha_{n, n}^{(s)}$ are interlaced. Thus for $i>N(n, s)$ the constants $B^{(s)}(i, m ; p, n)$ are all of the same sign because $m>M^{*}(n, s) \geqq M(n, s)$ implies $\phi_{m}^{(s)}(x)$ has exactly $N(n, s)$ zeros in $\left(0, \alpha_{n, n}^{(s)}\right)$. But by inspection $B^{(s)}(m, m ; p, n)$ is positive, so $B^{(s)}(i, m ; p, n)$ is positive for $N(n, s)<i \leqq m$; and the corollary follows. Q.E.D.

Our next objective is a Mittag-Leffler expansion for the meromorphic function $x^{p} K^{(s)}(x) \cdots K^{(s+n)}(x)$. Since we will be working with fixed $p, n$, and $s$ satisfying $0 \leqq p \leqq n, s \geqq 0$, we adopt the convention of dropping these from much of our notation: i.e., $B^{(s)}(i, m ; p, n)=B(i, m)$.

THEOREM 4.5. For $0 \leqq p \leqq n$ and $s \geqq 0$ the meromorphic function $x^{p} K^{(s)}(x) \cdots$ $K^{(s+n)}(x)$ has a series expansion of the form

$$
x^{p} K^{(s)}(x) \cdots K^{(s+n)}(x)=\sum_{i=1}^{\infty} \frac{B(i)}{x-\alpha_{i}^{(s)}}
$$

where $B(i)>0$ for $i>N(n, s)$ and $\sum_{i=1}^{\infty} B(i) / \alpha_{i}<\infty$. 
Proof. By definition, for $j \geqq 0$,

$$
K^{(s+j)}(x)=\lim _{m \rightarrow \infty} \frac{\phi_{m-j-1}^{(s+j+1)}(x)}{\phi_{m-j}^{(s+j)}(x)}
$$

where the convergence is uniform on compact sets bounded away from poles. Hence by using Corollary 4.4 we have

$$
\begin{aligned}
x^{p} K^{(s)}(x) \cdots K^{(s+n)}(x) & =\lim _{m \rightarrow \infty} x^{p} \frac{\phi_{m-1}^{(s+1)}(x)}{\phi_{m}^{(s)}(x)} \frac{\phi_{m-2}^{(s+2)}(x)}{\phi_{m-1}^{(s+1)}(x)} \cdots \frac{\phi_{m-n+1}^{(s+n+1)}(x)}{\phi_{m-n}^{(s+n)}(x)} \\
& =\lim _{m \rightarrow \infty} \frac{x^{p} \phi_{m-n-1}^{(s+n+1)}(x)}{\phi_{m}^{(s)}(x)} \\
& =\lim _{m \rightarrow \infty}\left\{\sum_{i=1}^{m} \frac{B(i, m)}{x-\alpha_{i, m}}\right\} .
\end{aligned}
$$

Now, by Hurwitz's theorem, $\alpha_{i, m}^{(s)} \rightarrow \alpha_{i}^{(s)}$ as $m \rightarrow \infty$ because $\alpha_{i}^{(s)}$ is the $i$ th pole of $K^{(s)}(x)$ and $K^{(s)}(x)=\lim _{m \rightarrow \infty} \phi_{m-1}^{(s+1)}(x) / \phi_{m}^{(s)}(x)$. Thus if we let $B(i)=B^{(s)}(i ; p, n)$ be the residue of $x^{p} K^{(s)}(x) \cdots K^{(s+n)}(x)$ at the pole $x=\alpha_{i}^{(s)}$ then, because of the uniform convergence, $B(i, m) \rightarrow B(i)$ as $m \rightarrow \infty$. Therefore

$$
\lim _{m \rightarrow \infty} \sum_{i=1}^{m} \frac{B(i, m)}{x-\alpha_{i, m}^{(s)}}=\sum_{i=1}^{N} \frac{B(i)}{x-\alpha_{i}}+\lim _{m \rightarrow \infty} \sum_{N+1}^{m} \frac{B(i, m)}{x-\alpha_{i, m}}
$$

where the last limit must also converge uniformly on compact sets bounded away from poles. Now for $m>M^{*}(n, s)$, each of the residues in the sum

$$
\sum_{N+1}^{m} B(i, m) /\left(x-\alpha_{i, m}\right)
$$

is positive by Corollary 4.4. Hence the sum $\sum_{N+1}^{m} B(i, m) /\left(x-\alpha_{i, m}\right)$ represents a rational function whose zeros and poles are interlaced on the positive real axis. This means we can apply the Montel theorem which we have been using. This is Theorem 3.3 above and applying it gives

$$
\lim _{m \rightarrow \infty} \sum_{i=N+1}^{m} \frac{B(i, m)}{x-\alpha_{i, m}}=-B+\sum_{N+1}^{\infty} \frac{B(i)}{x-\alpha_{i}}
$$

where $B(i)>0, i \geqq N+1$, and $\sum_{N+1}^{\infty} B(i) / \alpha_{i}<\infty$. Thus

Next, by Corollary 3.7

$$
x^{p} K^{(s)}(x) \cdots K^{(s+n)}(x)=-B+\sum_{i=1}^{\infty} \frac{B(i)}{x-\alpha_{i}}
$$

$$
\lim _{y \rightarrow \infty}(i y)^{p} K^{(s)}(i y) \cdots K^{(s+n)}(i y)=0 .
$$

Thus if we can show that we also have

$$
\lim _{y \rightarrow \infty}(i y)^{p} K^{(s)}(i y) \cdots K^{(s+n)}(i y)=-B,
$$


then $B=0$ and the theorem will be proven. But $(*)$ follows easily because given any $\varepsilon>0$, since $\sum_{1}^{\infty} B(i) / \alpha_{i}<\infty$, we can find a $K$ such that $\sum_{k=K+1}^{\infty} B(k) / \alpha_{k}<\varepsilon / 2$. We can then choose $y$ so $(1 /|y|) \sum_{k=1}^{K} B(k)<\varepsilon / 2$. Hence

$$
\begin{aligned}
\left|(i y)^{p} K^{(s)}(i y) \cdots K^{(s+n)}(i y)+B\right| & =\left|\sum_{k=1}^{\infty} B(k) /\left(i y-\alpha_{k}\right)\right| \\
& \leqq \sum_{k=1}^{K} \frac{B(k)}{\left|i y-\alpha_{k}\right|}+\sum_{k=K+1}^{\infty} \frac{B(k)}{\alpha_{k}} \frac{1}{\left|1-i y / \alpha_{k}\right|} \\
& \leqq(1 /|y|) \sum_{k=1}^{K} B(k)+\sum_{k=K+1}^{\infty} B(k) / \alpha_{k} \\
& <\varepsilon / 2+\varepsilon / 2=\varepsilon,
\end{aligned}
$$

and since $\varepsilon$ was arbitrary, this completes the proof. Q.E.D.

Before applying the above results in the construction of $\psi^{(s)}(x)$ we obtain some additional information about the sums of the residues $B(i)$ and $B(i, m)$, respectively.

LEMMA 4.6. For $s \geqq 0,0 \leqq p \leqq n$, and $m \geqq n+1$ we have

$$
\sum_{i=1}^{m} B(i, m)=\delta_{n, p}
$$

Proof. By the definition of $B(i, m)$

$$
x^{p+1} \frac{\phi_{m-n-1}^{(s+n+1)}(x)}{\phi_{m}^{(s)}(x)}=\sum_{i=1}^{m} B(i, m)\left\{\frac{x}{x-\alpha_{i, m}}\right\}
$$

Now let $x=1 / w$ and recall that $\phi_{m}^{(s)}(x)$ is monic. Thus $F_{m}^{(s)}(w)=w^{m} \phi_{m}^{(s)}(1 / w)$ satisfies $F_{m}^{(s)}(0)=1$. Therefore,

$$
\frac{w^{-p-1} \phi_{m-n-1}^{(s+n+1)}(1 / w)}{\phi_{m}^{(s)}(1 / w)}=\sum_{i=1}^{m} B(i, m)\left\{\frac{1}{1-w \alpha_{i, m}}\right\}
$$

so

$$
\frac{w^{n-p} F_{m-n-1}^{(s+n+1)}(w)}{F_{m}^{(s)}(w)}=\sum_{i=1}^{m} B(i, m)\left\{\frac{1}{1-w \alpha_{i, m}}\right\}
$$

And setting $w=0$ gives

$$
\delta_{n, p}=\sum_{i=1}^{m} B(i, m)
$$

THEOREM 4.7. For $s \geqq 0,0 \leqq p \leqq n$, we have

$$
\sum_{k=1}^{\infty} B(k)=\delta_{n, p}
$$

Proof. Since $B(k, m) \rightarrow B(k)$ as $m \rightarrow \infty$, we know that $B(k)$ is nonnegative for 
$k>N(n, s)$. Thus $\left\{\sum_{k=1}^{K} B(k)\right\}_{K=N(n, s)}^{\infty}$ is an increasing sequence, and it will converge if it is bounded. But by Lemma 4.6, for each natural number $K$ we have

$$
\left|\sum_{k=1}^{K} B(k)\right|=\left|\lim _{m \rightarrow \infty} \sum_{k=1}^{K} B(k, m)\right| \leqq \delta_{n, p} .
$$

Thus $\sum_{k=1}^{\infty} B(k)$ converges, and we need only show its sum is $\delta_{n, p}$. Now from Corollary 3.7

$$
\lim _{y \rightarrow \infty}(i y)^{p+1} K^{(s)}(i y) \cdots K^{(s+n)}(i y)=\delta_{n, p}
$$

and since

$$
x^{p+1} K^{(s)}(x) \cdots K^{(s+n)}(x)=\sum_{k=1}^{\infty} B(k) \frac{x}{x-\alpha_{k}}
$$

this gives

$$
\lim _{y \rightarrow \infty} \sum_{k=1}^{\infty} B(k)\left\{\frac{i y}{i y-\alpha_{k}}\right\}=\delta_{n, p}
$$

Finally, since $\sum_{k=1}^{\infty} B(k)$ converges,

$$
\lim _{y \rightarrow \infty} \sum_{k=1}^{\infty} B(k)\left\{\frac{i y}{i y-\alpha_{k}}\right\}=\sum_{k=1}^{\infty} B(k)
$$

and the theorem is proven. Q.E.D.

We now begin with the actual construction of $\psi^{(s)}(x)$. First we need an important recursion relationship which says that for $m \geqq 2, s \geqq 0$,

$$
\phi_{m}^{(s)}(x)=\left(x-a_{s}\right) \phi_{m-1}^{(s+1)}(x)-b_{1+s} \phi_{m-2}^{(s+2)}(x) .
$$

This follows easily by induction and the proof is omitted. Dividing through (IV-C) by $\phi_{m-1}^{(s+1)}(x)$ and letting $m \rightarrow \infty$, we obtain

$$
1 / K^{(s)}(x)=\left(x-a_{s}\right)-b_{1+s} K^{(s+1)}(x) .
$$

Combining this with the basic recurrence relationship (I-A) we obtain

$$
\phi_{n}^{(s)}(x) \cdot K^{(s)}(x)-\phi_{n-1}^{(s+1)}(x)=b_{1+s} \cdot K^{(s)}(x)\left\{\phi_{n-1}^{(s+1)} \cdot K^{(s+1)}(x)-\phi_{n-2}^{(s+2)}(x)\right\} .
$$

Since the expression in braces is just the left side with a change of index, we iterate and after multiplying by $x^{p}$ we obtain

$$
x^{p} \phi_{n}^{(s)}(x) \cdot K^{(s)}(x)-x^{p} \phi_{n-1}^{(s+1)}(x)=x^{p}\left\{\prod_{i=1}^{n} b_{i+s}\right\}\left\{\prod_{i=0}^{n} K^{(s+i)}(x)\right\} .
$$

We now consider this to be a relationship in the complex plane and after dividing by $2 \pi i$ we integrate both sides about the circle $|x|=R>0$. Thus

$$
\frac{1}{2 \pi i} \int_{|x|=R} x^{p} \phi_{n}^{(s)}(x) K^{(s)}(x) d x
$$

$$
=\left\{\prod_{i=1}^{n} b_{i+s}\right\} \frac{1}{2 \pi i} \int_{|x|=R} x^{p} K^{(s)}(x) \cdots K^{(s+n)}(x) d x .
$$


This leads to

THEOREM 4.8. If $0 \leqq p \leqq n, s \geqq 0$, then

$$
\lim _{R \rightarrow \infty} \frac{1}{2 \pi i} \int_{|x|=R} x^{p} \phi_{n}^{(s)}(x) K^{(s)}(x) d x=\delta_{n, p}\left\{\prod_{i=1}^{n} b_{i+s}\right\}
$$

Proof. In (IV-F) we replace $x^{p} K^{(s)}(x) \cdots K^{(s+n)}(x)$ by $\sum_{k=1}^{\infty} B(k) /\left(x-\alpha_{k}\right)$. This gives

$$
\frac{1}{2 \pi i} \int_{|x|=R} x^{p} \phi_{n}^{(s)}(x) K^{(s)}(x) d x=\left\{\prod_{i=1}^{n} b_{i+8}\right\} \frac{1}{2 \pi i} \int_{|x|=R} \sum_{k=1}^{\infty} B(k) /\left(x-\alpha_{k}\right) d x .
$$

Now if we let $K(R)$ represent the number of poles $\alpha_{k}$ which are in the disc $\{x:|x| \leqq R\}$ and then integrate the integral on the right term-by-term, we obtain

$$
\frac{1}{2 \pi i} \int_{|x|=R} x^{p} \phi_{n}^{(s)}(x) K^{(s)}(x) d x=\left\{\prod_{i=1}^{n} b_{i+s}\right\} \sum_{k=1}^{K(R)} B(k) .
$$

Finally, $K(R) \rightarrow \infty$ as $R \rightarrow \infty$, so by Theorem 4.7

$$
\begin{aligned}
\lim _{R \rightarrow \infty} \frac{1}{2 \pi i} \int_{|x|=R} x^{p} \phi_{n}^{(s)}(x) K^{(s)}(x) d x & =\left\{\prod_{i=1}^{n} b_{i+s}\right\} \lim _{R \rightarrow \infty} \sum_{k=1}^{K(R)} B(k) \\
& =\left\{\prod_{i=1}^{n} b_{i+s}\right\} \delta_{n, p} .
\end{aligned}
$$

We now give our main result.

THEOREM 4.9. Let the sequences $\left\{a_{i}\right\}_{0}^{\infty}$ and $\left\{b_{i}\right\}_{1}^{\infty}$ satisfy the following conditions:

(1) $a_{n} \rightarrow \infty$ as $n \rightarrow \infty$.

(2) $b_{n}>0$ for each $n$ and

$$
\limsup _{n \rightarrow \infty} \frac{b_{n}}{a_{n} a_{n-1}}=L<1 / 4 .
$$

Then if $K^{(s)}(x)$ and $\psi^{(s)}(x)$ are defined by (5) and (6) of $\$ 1.1$, we have

(i) $K^{(s)}(x)$ is a meromorphic function and has a representation of the form

$$
K^{(s)}(x)=\sum_{k=1}^{\infty} A_{k}^{(s)} /\left(x-\alpha_{k}^{(s)}\right)
$$

where $A_{k}^{(s)}>0$ for each $k, \sum_{1}^{\infty} A_{k}^{(s)}=1$, and $\alpha_{1}^{(s)}<\alpha_{2}^{(s)}<\cdots$.

(ii) $\psi^{(s)}(x)$ can be chosen to be a jump function with a jump of $A_{k}^{(s)}$ at $x=\alpha_{k}^{(s)}$, $k=1,2, \ldots$, while being constant on each of the intervals $\left(-\infty, \alpha_{1}^{(s)}\right),\left(\alpha_{1}^{(s)}, \alpha_{2}^{(s)}\right)$, $\left(\alpha_{2}^{(s)}, \alpha_{3}^{(s)}\right), \ldots$

Proof. (i) is just Corollary 3.6 together with Theorem 4.7, so we need only prove (ii). Thus we let $\psi^{(s)}(x)$ be defined by the following:

(1) $\psi^{(s)}(-\infty)=0$;

(2) $\psi^{(s)}(x)$ is constant on each of the intervals $\left(-\infty, \alpha_{1}^{(s)}\right),\left(\alpha_{1}^{(s)}, \alpha_{2}^{(s)}\right),\left(\alpha_{2}^{(s)}, \alpha_{3}^{(s)}\right), \ldots$; 
(3) $\psi^{(s)}\left(\alpha_{k}^{(s)}\right)-\psi^{(s)}\left(\alpha_{k}^{(s)}-0\right)=A^{(s)}, k=1,2,3, \ldots$;

then

$$
\int_{-\infty}^{+\infty} x^{p} \phi_{n}^{(s)}(x) d \psi^{(s)}(x)=\sum_{k=1}^{\infty}\left[\alpha_{k}^{(s)}\right]^{p} \phi_{n}^{(s)}\left(\alpha_{k}^{(s)}\right) A_{k}^{(s)}
$$

Next if we substitute $\sum_{k=1}^{\infty} A_{k}^{(s)} /\left(x-\alpha_{k}^{(s)}\right)$ for $K^{(s)}(x)$ in formula (IV-G), we obtain

$$
\lim _{R \rightarrow \infty} \frac{1}{2 \pi i} \int_{|x|=R} x^{p} \phi_{n}^{(s)}(x)\left\{\sum_{k=1}^{\infty} \frac{A_{k}^{(s)}}{x-\alpha_{k}^{(s)}}\right\} d x=\left\{\prod_{i=1}^{n} b_{i+s}\right\} \delta_{n, p} .
$$

Hence, by integrating term-by-term we have

$$
\lim _{R \rightarrow \infty} \sum_{k=1}^{K(R)}\left[\alpha_{k}^{(s)}\right]^{p} \phi_{n}^{(s)}\left(\alpha_{k}^{(s)}\right) A_{k}^{(s)}=\left\{\prod_{i=1}^{n} b_{i+s}\right\} \delta_{n, p},
$$

so

$$
\sum_{K=1}^{\infty}\left[\alpha_{k}^{(s)}\right]^{p} \phi_{n}^{(s)}\left(\alpha_{k}^{(s)}\right) A_{k}^{(s)}=\left\{\prod_{i=1}^{n} b_{i+s}\right\} \delta_{n, p}
$$

and

$$
\int_{-\infty}^{+\infty} x^{p} \phi_{n}^{(s)}(x) d \psi^{(s)}(x)=\left\{\prod_{i=1}^{n} b_{i+s}\right\} \delta_{n, p}
$$

Therefore, $\psi^{(s)}(x)$ is a distribution function for the polynomials $\left\{\phi_{n}^{(s)}\right\}_{-1}^{\infty}$ and the theorem is proven. Q.E.D.

We now modify assumption (1) of Theorem 4.9 by having $a_{n} \rightarrow-\infty$ as $n \rightarrow \infty$. This gives:

COROLlaRY 4.10. If the real sequences $\left\{a_{n}\right\}_{0}^{\infty}$ and $\left\{b_{n}\right\}_{1}^{\infty}$ satisfy the conditions:

(1*) $a_{n} \rightarrow-\infty$ as $n \rightarrow \infty$.

(2*) $b_{n}>0$ for each $n$ and

$$
\limsup _{n \rightarrow \infty}\left(b_{n} /\left(a_{n} a_{n-1}\right)\right)=L<1 / 4 ;
$$

then

(i*) $K^{(s)}(x)=\sum_{k=1}^{\infty} A_{k}^{(s)} /\left(x-\alpha_{k}^{(s)}\right)$ where $A_{k}^{(s)}>0$ for each $k, \sum_{1}^{\infty} A_{k}^{(s)}=1$ and $-\infty<\cdots<\alpha_{3}^{(s)}<\alpha_{2}^{(s)}<\alpha_{1}^{(s)}$.

$\left(\mathrm{ii}^{*}\right) \psi^{(s)}(x)$ can be chosen to be a jump function which is constant on each of the intervals $\left(\alpha_{1}^{(s)}, \infty\right),\left(\alpha_{i+1}^{(s)}, \alpha_{i}^{(s)}\right), i=1,2, \ldots$, and has a jump $A_{k}^{(s)}$ at $x=\alpha_{k}^{(s)}, k=1,2,3, \ldots$

Proof. By definition, for each fixed $s \geqq 0$, the polynomials $\left\{\phi_{n}^{(s)}\right\}_{n=1}^{\infty}$ satisfy the triple recurrence formula

$$
\begin{aligned}
\phi_{n+1}^{(s)}(x) & =\left(x-a_{n}^{(s)}\right) \phi_{n}^{(s)}(x)-b_{n}^{(s)} \phi_{n-1}^{(s)}(x) \quad(n \geqq 0), \\
\phi_{0}^{(s)}(x) & =1, \phi_{-1}^{(s)}(x)=0 .
\end{aligned}
$$

Hence the polynomial set $(-1)^{n} \phi_{n}^{(s)}(-x)$ satisfies the relationship

$$
(-1)^{(n+1)} \phi_{n+1}^{(s)}(-x)=\left(x+a_{n}^{(s)}\right)(-1)^{n} \phi_{n}^{(s)}(-x)-b_{n}^{(s)}(-1)^{n-1} \phi_{n-1}^{(s+1)}(x)
$$


$(n \geqq 0)$ where we again set $\phi_{0}^{(s)}(x)=1, \phi_{-1}^{(s)}=0$. Thus the set $(-1)^{n} \phi_{n}^{(s)}(-x)$ is associated with the sequence $\left\{-a_{n}\right\}_{0}^{\infty}$ and $\left\{b_{n}\right\}_{1}^{\infty}$. But these sequences satisfy the hypothesis of Theorem 4.9, so if we let $K_{1}^{(s)}(x)$ and $\psi_{1}^{(s)}(x)$ be associated with them, then

(i) $K_{1}^{(s)}(x)=\sum_{k=1}^{\infty} A_{k}^{(s)} /\left(x-\beta_{k}^{(s)}\right)$ where $A_{k}^{(s)}>0$ for each $k, \sum_{k=1}^{\infty} A_{k}^{(s)}=1$, and $\beta_{1}^{(s)}<\beta_{2}^{(s)}<\cdots$;

(ii) $\psi_{1}^{(s)}(x)$ can be chosen to be a jump function which is constant on each of the intervals $\left(-\infty, \beta_{1}^{(s)}\right),\left(\beta_{i}^{(s)}, \beta_{i+1}^{(s)}\right), i=1,2, \ldots$, and has jump $A_{k}^{(s)}$ at $x=\beta_{k}^{(s)}$. Now

$$
(-1) K^{(s)}(-x)=\lim _{m \rightarrow \infty} \frac{(-1) \phi_{m-1}^{(s+1)}(-x)}{\phi_{m}^{(s)}(-x)}=\lim _{m \rightarrow \infty} \frac{(-1)^{m-1} \phi_{m-1}^{(s+1)}(-x)}{(-1)^{m} \phi_{m}^{(s)}(-x)}=K_{1}^{(s)}(x),
$$

so

$$
K^{(s)}(x)=(-1) K_{1}^{(s)}(-x)=\sum_{k=1}^{\infty} A_{k}^{(s)} /\left(x+\beta_{k}^{(s)}\right)
$$

Hence, we see that by taking $\alpha_{k}^{(s)}=-\beta_{k}^{(s)}, k=1,2, \ldots$, conclusion (i*) follows.

Next, since $\psi_{1}^{(s)}(x)$ is a distribution function for the monic polynomial set $\left\{(-1)^{n} \phi_{n}^{(s)}(x)\right\}_{n=-1}^{\infty}$, we have for $0 \leqq p \leqq n$, and some $k_{n} \neq 0, n=0,1, \ldots$

$$
k_{n} \cdot \delta_{n, p}=\int_{-\infty}^{+\infty} x^{p}(-1)^{n} \phi_{n}^{(s)}(-x) d \psi_{1}^{(s)}(x)
$$

Replacing $x$ by $-x$ gives

Therefore,

$$
k_{n} \cdot \delta_{n, p}=(-1)^{n+p} \int_{-\infty}^{+\infty} x^{p} \phi_{n}^{(s)}(x)(-1) d \psi_{1}^{(s)}(-x)
$$

$$
\begin{aligned}
\int_{-\infty}^{+\infty} x^{p} \phi_{n}^{(s)}(x)\left\{(-1) d \psi_{1}^{(s)}(-x)\right\} & =(-1)^{n+p} \cdot k_{n} \delta_{n, p} \\
& =k_{n}^{\prime} \cdot \delta_{n, p}
\end{aligned}
$$

where $k_{n}^{\prime} \neq 0, n=0,1,2, \ldots$, so if we let $\psi^{(s)}(x)=(-1) \psi_{1}^{(s)}(-x)$, then $\psi^{(s)}(x)$ is a distribution function for $\left\{\phi_{n}^{(s)}(x)\right\}$. Therefore, (ii*) follows since $\psi_{1}^{(s)}(x)$ satisfies (ii). Q.E.D.

5. Applications. In this section we use the results of $\S 4$ to answer some questions about a known polynomial set. We shall also obtain some information about quotients of Bessel functions, where these functions are considered to be functions of their order. The polynomial set which we consider is the set of modified Lommel polynomials. We follow Dickinson, [10, p. 120], in defining these polynomials as follows: $R_{-1}(\nu, x)=0, R_{0}(\nu, x)=1$ and for $n \geqq 0$

$$
R_{n+1}(\nu, x)=(2 / x)(\nu+n) R_{n}(\nu, x)-R_{n-1}(\nu, x) .
$$

Thus for each real, nonzero value of $x,\left\{R_{n}(\nu, x)\right\}_{-1}^{\infty}$ is a set of real polynomials in the variable $\nu$. Now, in [10, p. 121], Dickinson asked for the construction of a distribution function in $\nu$ for these polynomials. Our results allow us to carry out 
this construction. First, since the set $\left\{R_{n}(\nu, x)\right\}$ is not monic, we shall work with the polynomial set $\left\{P_{n}(\nu, x)\right\}_{-1}^{\infty}$ defined by $P_{-1}(\nu, x)=0, P_{0}(\nu, x)=1$ and for $n \geqq 1$, $P_{n}(\nu, x)=(2 x)^{-n} \cdot R_{n}(\nu, 1 / x)$. Then from $(\mathrm{V}-\mathrm{A})$ we have

$$
P_{n+1}(\nu, x)=(\nu+n) P_{n}(\nu, x)-(2 x)^{-2} \cdot P_{n-1}(\nu, x)
$$

where $n \geqq 0$. This means that the polynomial set $\left\{P_{n}(\nu, x)\right\}_{-1}^{\infty}$ is associated with the sequences $\left\{a_{n}=-n\right\}_{0}^{\infty}$ and $\left\{b_{n}=(2 x)^{-2}\right\}_{1}^{\infty}$. But for $x \neq 0$ these sequences satisfy the hypothesis of Corollary 4.10 and hence we have the following theorem.

THEOREM 5.1. For each real $x \neq 0$ and nonnegative integer $s$, we have

(i) the continued fraction

$$
\begin{aligned}
K^{(s)}(\nu, x) & =\frac{1 \mid}{\mid+s}-\frac{(2 x)^{-2} \mid}{\mid+s+1}-\frac{(2 x)^{-2} \mid}{\mid+s+2}-\cdots \\
& =\lim _{n \rightarrow \infty} \frac{P_{n-1}^{(s+1)}(\nu, x)}{P_{n}^{(s)}(\nu, x)}
\end{aligned}
$$

converges to a meromorphic function which has an expansion of the form

$$
K^{(s)}(\nu, x)=\sum_{k=1}^{\infty} A_{k}^{(s)} /\left(\nu-\nu_{k}^{(s)}(x)\right)
$$

where $A_{k}^{(s)}(x)>0$ for each $k, \sum_{k=1}^{\infty} A_{k}^{(s)}(x)=1$, and $-\infty<\cdots<\nu_{2}^{(s)}(x)<\nu_{1}^{(s)}(x)<\infty$. Also $s \geqq 1$ and $|x| \geqq 1$ implies $\nu_{1}^{(s)}(x) \leqq 0$.

(ii) The polynomials $P_{n}(\nu, x), n=0,1,2, \ldots$ are orthogonal with respect to a distribution function $\psi^{(s)}(\nu, x)$ that is constant on each of the intervals $\left(\nu_{1}^{(s)}(x), \infty\right),\left(\nu_{i+1}^{(s)}(x)\right.$, $\left.\nu_{i}^{(s)}(x)\right), i=1,2, \ldots$ and which has a jump of height $A_{k}^{(s)}(x)$ at $\nu=\nu_{k}^{(s)}(x), k=1,2, \ldots$ Moreover $\psi^{(s)}(\nu, x)$ is essentially unique.

Proof. Corollary 4.10 gives all the results except the condition on $\nu_{1}^{(s)}(x)$ and the uniqueness of $\psi^{(s)}(\nu, x)$. The condition on $\nu_{1}^{(s)}(x)$ follows by considering $(-1) \psi^{(s)}$ $\cdot(-\nu, x)$ and applying Theorem 2.1. This theorem applies because $s \geqq 1$ and $|x| \geqq 1$ implies $\left\{(2 x)^{-2} /\left(a_{n+s} \cdot a_{n+s-1}\right)\right\}$ is a chain sequence. This together with $a_{n+s}>0$, $n=0,1,2, \ldots$ implies $-\nu_{1}^{(s)}(x) \geqq 0$ or $\nu_{1}^{(s)}(x) \leqq 0$.

The essential uniqueness of $\psi^{(s)}(\nu, x)$ is a result of Carleman's criteria, [16, p. 59]. This says $\psi^{(s)}(\nu, x)$ is essentially unique if $\sum_{1}^{\infty}\left(b_{n}\right)^{-1 / 2}=\infty$. In our case $b_{n}=(2 x)^{-2}$ so the result follows. Q.E.D.

Next, since the polynomials $P_{n}(\nu, x)$ are just a monic version of $R_{n}(\nu, 1 / x)$ we see that the modified Lommel polynomials are orthogonal over a denumerable set consisting of the poles of the meromorphic function

$$
(1 / 2 x) K^{(s)}(\nu, x)=\lim _{n \rightarrow \infty}\left\{R_{n-1}^{(s+1)}(\nu, 1 / x) / R_{n}^{(s)}(\nu, 1 / x)\right\} .
$$

We now examine this function $K^{(s)}(\nu, x)$ more closely and show that it is just a quotient of Bessel functions. We first state a lemma due to Watson, [20, p. 302]. 
LEMMA 5.2. If $J(\nu, z)$ represents the Bessel function of order $\nu$ and argument $z$, and if $\Gamma(w)$ is the gamma function, then

$$
\lim _{m \rightarrow \infty} \frac{(z / 2)^{v+m} R_{m}(\nu+1, z)}{\Gamma(\nu+m+1)}=J(\nu, z) .
$$

We also need a relationship between $R_{m}^{(s)}(\nu, z)$ and $R_{m}^{(s-n)}(\nu+n, z)$. We prove this as another lemma.

LEMMA 5.3. For integers $s \geqq n \geqq 0$ and $m \geqq 0$ we have $R_{m}^{(s)}(\nu, z)=R_{m}^{(s-n)}(\nu+n, z)$.

Proof. We have from (V-A) and our definition of $R_{m}^{(s)}(\nu, z)$ that

$$
R_{m+1}^{(s)}(\nu, z)=(\nu+m+s) R_{m}^{(s)}(\nu, z)-(2 x)^{-2} R_{m-1}^{(s)}(\nu, z) .
$$

Thus,

$$
R_{m+1}^{(s-n)}(\nu+n, z)=(\nu+m+s) R_{m}^{(s-n)}(\nu+n, z)-(2 x)^{-2} R_{m-1}^{(s-n)}(\nu+n, z) .
$$

Also for $s-n \geqq 0, R_{-1}^{(s-n)}(\nu+n, z)=0$ and $R_{0}^{(s-n)}(\nu+n, z)=1$. Hence we see that the polynomial sequences $\left\{R_{m}^{(s)}(\nu, z)\right\}$ and $\left\{R_{m}^{(s-n)}(\nu+n, z)\right\}$ satisfy the same triple recurrence formula and have the same initial elements. Therefore $R_{m}^{(s)}(\nu, z)=$ $R_{m}^{(s-n)}(\nu+n, z)$ as was to be shown. Q.E.D.

We now use these two lemmas to obtain our results about Bessel functions.

THEOREM 5.4. For each integer $s \geqq 0$ and real $x \neq 0$ we have

$$
(1 / 2 x) K^{(s)}(\nu, x)=\frac{J(\nu+s+1,1 / x)}{J(\nu+s, 1 / x)}
$$

Proof. From part (i) of Theorem 5.1 we have

$$
\begin{aligned}
(1 / 2 x) K^{(s)}(\nu, x) & =\lim _{m \rightarrow \infty} \frac{P_{m-1}^{(s+1)}(\nu, x)}{(2 x) P_{m}^{(s)}(\nu, x)} \\
& =\lim _{m \rightarrow \infty} \frac{(2 x)^{-m+1} R_{m-1}^{(s+1)}(\nu, 1 / x)}{(2 x)^{-m+1} R_{m}^{(s)}(\nu, 1 / x)}
\end{aligned}
$$

Thus using Lemmas 5.2 and 5.3 this gives

$$
\begin{aligned}
(1 / 2 x) K^{(s)}(\nu, x) & =\lim _{m \rightarrow \infty} \frac{R_{m-1}(\nu+s+1,1 / x)}{R_{m}(\nu+s, 1 / x)} \\
& =\lim _{m \rightarrow \infty} \frac{(1 / 2 x)^{+m+s-1} R_{m-1}(\nu+s+1,1 / x) / \Gamma(\nu+m+s)}{(1 / 2 x)^{+m+s-1} R_{m}(\nu+s, 1 / x) / \Gamma(\nu+m+s)} \\
& =\frac{J(\nu+s+1,1 / x)}{J(\nu+s, 1 / x)} .
\end{aligned}
$$

COROLlaRY 5.5. As functions of their order $\nu$, and for real $z$, the Bessel functions $J(\nu+1, z)$ and $J(\nu, z)$ have real, simple zeros which are interlaced on the interval $(-\infty, c)$ for some finite $c$. 
Proof. Since $K^{(0)}(\nu, 1 / z)=(z / 2) J(\nu+1, z) / J(\nu, z)$, the zeros and poles of $K^{(0)}(\nu, 1 / z)$ are just the zeros of $J(\nu+1, z)$ and the zeros of $J(\nu, z)$. Thus the result follows from the known properties of $K^{(0)}(\nu, 1 / z)$. Q.E.D.

COROLlaRY 5.6. For real $x \neq 0$

$$
\frac{J(\nu+1,1 / x)}{J(\nu, 1 / x)}=\sum_{k=1}^{\infty} \frac{A_{k}(x)}{\nu-\nu_{k}(x)}
$$

where $A_{k}(x)>0$ for $k=1,2, \ldots$ and $\sum_{k=1}^{\infty} A_{k}(x)=1$.

Proof. Combining Theorems 5.1 and 5.4 with $s=0$ gives the result. Q.E.D.

COROLlaRY 5.7. For each real $z_{0}$, there are a denumerable number of Bessel functions $J\left(v_{i}, z\right), i=1,2, \ldots$, which have a zero at $z_{0}$. Moreover $-\infty<\cdots<\nu_{2}$ $<\nu_{1}<\infty$.

Proof. This is just a restatement of the fact that $(z / 2) K^{(0)}(\nu, 1 / z)=J(\nu+1, z) / J(\nu, z)$ had denumerably many poles on the real axis for each real $z$. The conditions on the $\nu_{i}$ 's follow from Theorem 5.1. Q.E.D.

REMARK. An alternate proof of Corollary 5.7 and the fact that the zeros of $J(\nu, z)$ are real was given by Coulomb, [8, p. 297-302]. He also showed that these zeros $\nu_{i}(x)$ are asymptotic to the negative integers.

6. Acknowledgements. Thanks are due to Professor Jack Goldberg of The University of Michigan. Discussions with him and suggestions of his were a major source of inspiration for writing the thesis which led to this paper.

\section{BIBLIOGRAPHY}

1. N. I. Ahiezer, The classical moment problem, Oliver and Boyd, Edinburgh, 1965.

2. N. I. Ahiezer and M. G. Kreĭn, Some questions in the theory of moments, Transl. Math. Monographs, Vol. 2, Amer. Math. Soc., Providence, R. I., 1962.

3. L. Carlitz, On some polynomials of Tricomi, Boll. Un. Mat. Ital. (3) 13 (1958), 58-64.

4. T. S. Chihara, On recursive orthogonal polynomials, Proc. Amer. Math. Soc. 8 (1957), 899-905.

5. __ On kernel polynomials and related systems, Boll. Un. Mat. Ital. (3) 19 (1964), 451-459.

6. - Chain sequences and orthogonal polynomials, Trans. Amer. Math. Soc. 14 (1962), $1-16$.

7. _- On recursively defined orthogonal polynomials, Proc. Amer. Math. Soc. 16 (1965), 702-710.

8. M. J. Coulomb, Sur les zéros des fonctions de Bessel considérées comme fonctions de 1 ordre, Bull. Sci. Math. 60 (1936), 297-302.

9. D. J. Dickinson, On Bessel and Lommel polynomials, Proc. Amer. Math. Soc. 5 (1954), 946-956.

10. - On certain polynomials associated with orthogonal polynomials, Boll. Un. Mat. Ital. (3) 13 (1958), 116-124.

11. D. J. Dickinson, H. C. Pollak and G. H. Wannier, On a class of polynomials orthogonal over a denumerable set, Pacific J. Math. 6 (1956), 239-247. 
12. J. Favard, Sur les polynomes de Tchebicheff, C. R. Acad. Sci. Paris 200 (1935), 20522053.

13. E. Hille, Analytic function theory, Vol. II, Ginn, New York, 1962.

14. J. L. Goldberg, Some polynomials orthogonal over a denumerable set, Pacific J. Math. 15 (1965), 1171-1186.

15. N. Obrechkoff, Quelques classes de fonctions entières limites de polynomes et de fonctions méromorphes limites de fractions rationnelles, Actualités Sci. Ind. No. 891, Hermann, Paris, 1941.

16. J. A. Shohat and J. D. Tamarkin, The problem of moments, Math. Surveys No. 1, Amer. Math Soc., Providence, R. I., 1950.

17. G. Szegö, Orthogonal polynomials, Colloq. Publ., Vol. 23, Amer. Math. Soc., Providence, R. I., 1939.

18. E. B. Van Fleck, On the convergence of algebraic continued fractions whose coefficients have limiting values, Trans. Amer. Math. Soc. 17 (1904), 253-262.

19. H. S. Wall, Analytic theory of continued fractions, Van Nostrand, New York, 1948.

20. G. N. Watson, A treatise on the theory of Bessel functions, Cambridge Univ. Press, Cambridge, 1944.

THE UNIVERSITY OF Michigan,

ANN ARBor, Michigan

INDIANA UNIVERSITY,

BLOOMINGTON, INDIANA 